\title{
Serial Changes in Hamman's Sign Secondary to the Evolution of a Shallow Left-Sided Spontaneous Pneumothorax
}

\author{
Katsuhito Seki MD, Hiroyuki Watanabe MD, Ryo Morita MD, Kazuhiro Sato MD, \\ Mariko Asano MD, Takako Iino MD, Kenji Iino MD, and Hiroshi Ito MD
}

\section{Introduction}

Hamman's sign is a rare phenomenon caused by pneumomediastinum or pneumothorax. It was proposed for the first time in 1937 by Louis Hamman. It presents as a crunching or clicking noise in synchrony with the heartbeat and is best heard in the left lateral decubitus position. This case shows serial changes in Hamman's sign corresponding to pneumothorax progression, in which precordial clicking sounds were temporary and limited to the time period when the pneumothorax was small.

\section{Case Summary}

A 20-y-old male presented with sudden-onset precordial clicks. He did not complain of chest pain or dyspnea, although his mother said that the noise could be heard from a few meters away. Over the previous $2 \mathrm{y}$, he had similar repeated episodes, but each time, the sounds resolved spontaneously within 2 weeks. Cardiac auscultation revealed loud mid-systolic clicks that coincided with the heart sounds, rather than with respiration. Blood tests showed an elevated white blood cell count of $10.0 \times 10^{9}$ cells/L (reference interval of 4.0-9.0 $\times 10^{9}$ cells/L), but were otherwise normal. C-reactive protein was normal. Although much effort was expended seeking the cause of the mid-systolic clicks, including echocardiography, electrocardiography, and chest x-ray, the origin remained unclear. Two weeks later, the patient was referred for further

The authors are affiliated with the Department of Cardiovascular and Respiratory Medicine, Akita University School of Medicine, Akita, Japan.

The authors have disclosed no conflicts of interest.

Correspondence: Hiroyuki Watanabe MD, Department of Cardiovascular and Respiratory Medicine, Akita University School of Medicine, 1-1-1 Hondoh, Akita 010-8543, Japan. E-mail: hirow@doc.med.akita-u.ac.jp.

DOI: $10.4187 /$ respcare.03562 diagnostic workup in our hospital. Upon auscultation, we detected loud mid-systolic clicks that were not followed by a late systolic murmur (Fig. 1A). The only abnormality found was a left-sided pneumothorax on the chest x-ray (Fig. 1B). On re-evaluating the previous chest $\mathrm{x}$-ray, we noticed that a small pneumothorax had been missed. A diagnosis of pneumothorax with Hamman's sign, ${ }^{1}$ a socalled noisy pneumothorax, ${ }^{2}$ was made. Chest computed tomography showed air in the left pleural space, but not within the mediastinum or lung major fissure (Fig. 1C). Because of the shallow pneumothorax and lack of apparent symptoms, the patient was treated conservatively for 2 weeks. We simply monitored his condition by performing serial chest $\mathrm{X}$-rays. However, he developed mild breathlessness, and the pneumothorax increased in size during the follow-up observations (Fig. 2A). As the pneumothorax became progressively larger, the mid-systolic clicks became fainter and were audible only intermittently (Fig. 2B). We could no longer hear the clicks $8 \mathrm{~h}$ later (Fig. 3A). Partial resection of the left upper lobe was required because chest tube drainage was unsuccessful (Fig. 3B). The patient had an uneventful postoperative course.

\section{Discussion}

We report a case of a patient with a transient midsystolic click secondary to a small left-sided spontaneous pneumothorax. A mid-systolic click is commonly heard in cases of mitral valve prolapse, but a shallow pneumothorax is part of the differential diagnosis. Hamman's sign was coined in 1937 by Louis Hamman, who described a crackly, bubbly, crunchy sound that was heard over the precordium and synchronous with the heartbeat in a patient with pneumomediastinum. ${ }^{1}$ However, its etiology has remained unclear. The chest sounds described by Hamman might not represent a single phenomenon, as described originally. In 1955, Chapman proposed that the cause of Hamman's sign was not the pneumomediastinum, but rather a shallow left-sided pneumothorax that either co-existed or was present alone. ${ }^{3,4}$ Our case demonstrated that a pneumothorax alone can cause Hamman's sign, in agreement 

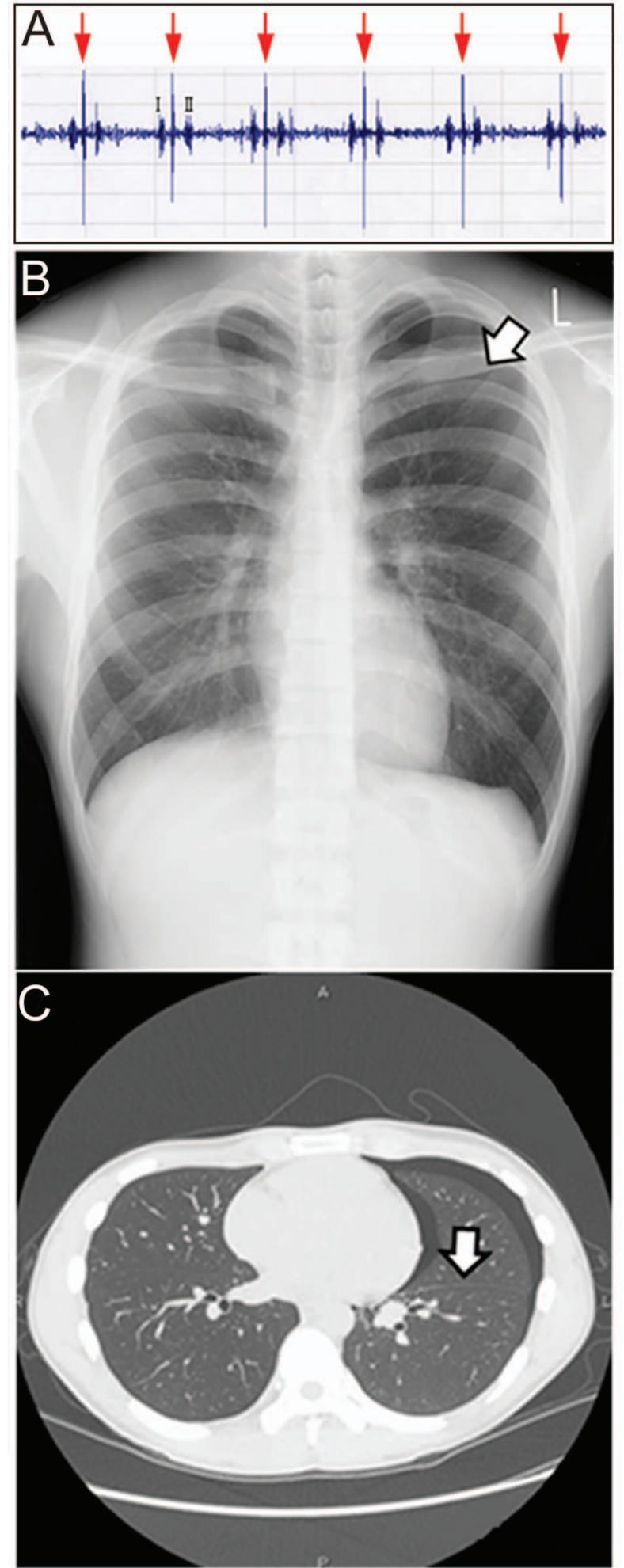

Fig. 1. A: Phonocardiogram at the first visit. Prominent mid-systolic clicks are recorded (arrows). B: Original chest $\mathrm{x}$-ray. Note a small left-sided pneumothorax. The arrow indicates the edge of the collapsed lung. C: Chest computed tomography shows the collapsed lung and air in the left pleural space. Note no air within the mediastinum and lung major fissure. The arrow indicates the lung major fissure.

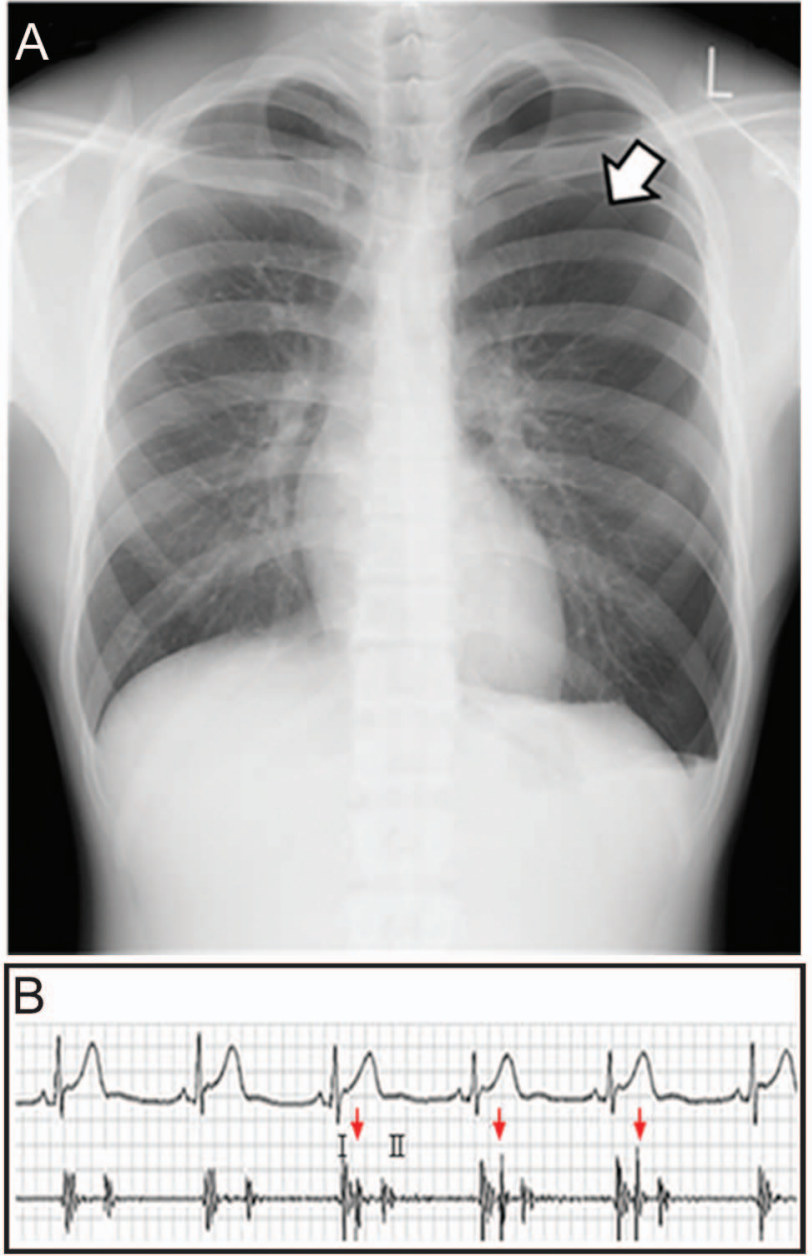

Fig. 2. A: Chest $x$-ray showing the pneumothorax deterioration. The arrow indicates the edge of the collapsed lung. B: Simultaneously recorded phonocardiogram of the mitral area and lead II of the echocardiogram. The mid-systolic clicks became fainter and were audible only intermittently (arrows).

with Chapman. Although reports have described Hamman's sign associated with a small left-sided pneumothorax, ${ }^{5,6}$ few reports have described serial changes in both chest X-rays and phonocardiograms. Our case showed serial changes in Hamman's sign corresponding to the progression of a pneumothorax, during which the clicks became progressively fainter as the pneumothorax grew. In 1992, Baumann and Sahn ${ }^{7}$ proposed a mechanism underlying Hamman's sign associated with pneumothorax: free pleural air is channeled cyclically through a lung major fissure, creating the sound. However, we could not demonstrate air within the lung major fissure on chest computed tomography or magnetic resonance imaging (data not shown) despite the audible mid-systolic clicks. These results suggest an alternative mechanism underlying the pathogenesis of Hamman's sign. It is likely that a variety 

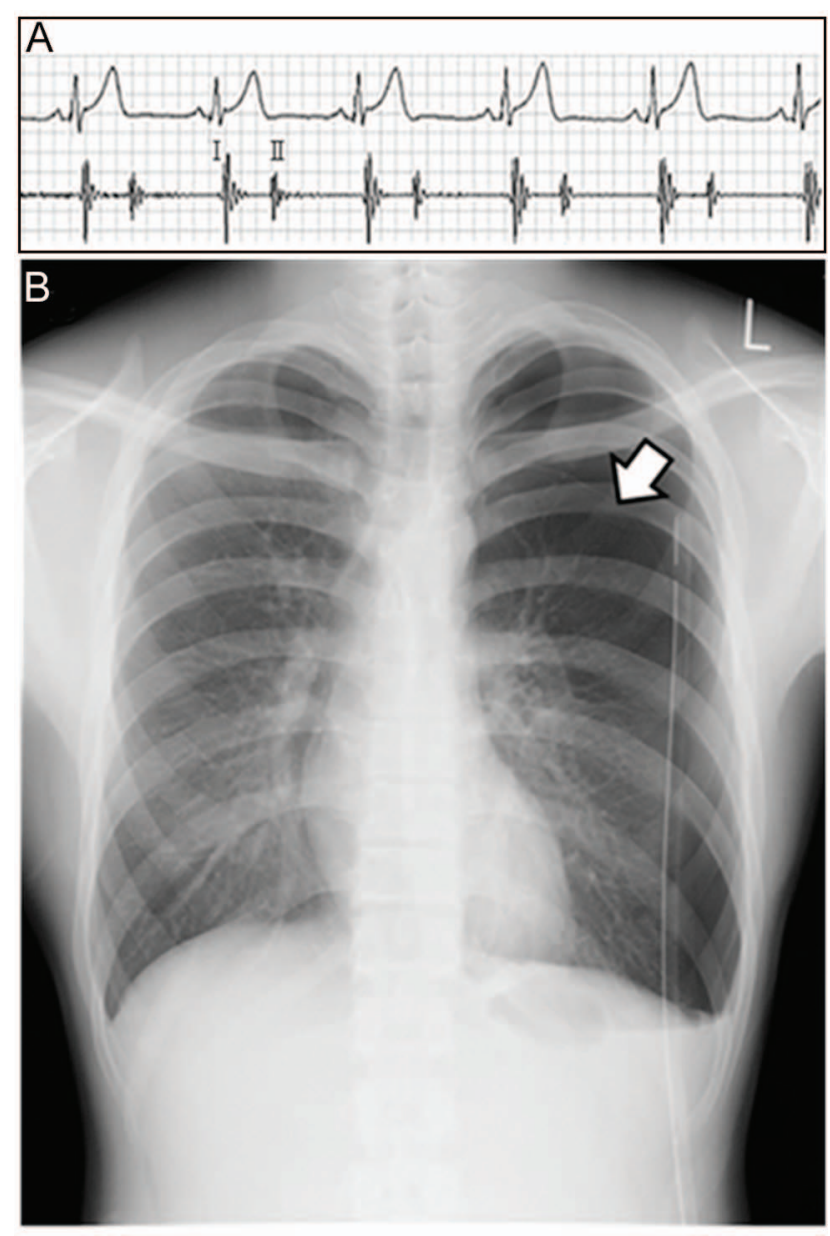

Fig. 3. A: Phonocardiogram 2 weeks after the first visit. No clicks are demonstrated. B: Chest $\mathrm{x}$-ray showing pneumothorax progression despite the chest tube drainage. The arrow indicates the edge of the collapsed lung.

of mechanisms contribute to the genesis of Hamman's sign.

Pneumothoraces with mild symptoms (particularly in smaller pneumothoraces) often represent a diagnostic challenge and might be missed by chest x-ray alone. Although Hamman's sign is rare, once it appears, a shallow leftsided pneumothorax or pneumomediastinum should be considered. An accurate and prompt diagnosis of pneumothorax can lead to early treatment and recovery.

\section{Teaching Points}

- Pneumothorax is rarely accompanied by a noise in the chest. Hamman's sign is one such chest finding, first described by Louis Hamman in 1937. The noise is characterized as a crunching, popping, crackling, or clicking sound synchronous with the heart sounds, rather than with respiration. It can occasionally be heard from several meters away without a stethoscope.

- Hamman's sign can be caused by pneumomediastinum, as well as by pneumothorax. Hamman's sign associated with pneumothorax appears only in the left-sided pneumothorax and is unique in that the clicks become progressively fainter as the pneumothorax grows and are altered by postural change (best heard in the left lateral decubitus position). However, the underlying mechanisms remain unknown.

- In our case, cardiac auscultation revealed loud mid-systolic clicks secondary to a spontaneous pneumothorax. A mid-systolic click is commonly heard in mitral valve prolapse, although a shallow pneumothorax is part of the differential diagnosis. Echocardiographic findings and other features of pneumothorax, including signs, symptoms, and medical imaging, are helpful in the differential diagnosis of mid-systolic clicks.

- Although Hamman's sign is rare, once it appears, a shallow left-sided pneumothorax or pneumomediastinum should be considered. An accurate and prompt diagnosis can lead to early treatment and recovery.

\section{REFERENCES}

1. Hamman L. Spontaneous interstitial emphysema of the lungs. Tr Assoc Am Physicians 1937;52:311-319.

2. Remmelts HH, Banga JD. Popping pneumothorax. Netherlands J Med 2010;68(4):187.

3. Chapman JS. Spontaneous irruption of air from the lung. I. Pneumomediastinum. Am J Med 1955;18(4):547-556.

4. Drury NE, Gnanapragasam JP. Recurrent noisy pneumothorax mimicking pericarditis. Int J Cardiol 2006;113(1):104-105.

5. Roelandt J, Willems J, van der Hauwaert LG, de Geest H. Clicks and sounds (whoops) in left-sided pneumothorax. Clinical and phonocardiographic study. Dis Chest 1969;56(1):31-36.

6. Scadding J, Wood P. Systole clicks due to left-sided pneumothorax. Lancet 1939;234(6067):1208-1211.

7. Baumann MH, Sahn SA. Hamman's sign revisited. Pneumothorax or pneumomediastinum? Chest 1992;102(4):1281-1282. 